\title{
Defining Safety Space for Functional Tongue Surgery in Korean Male Obstructive Sleep Apnea Syndrome Patients; Analysis on Spatial Relation of the Tongue and the Lingual Artery
}

\author{
Marn Joon Park ${ }^{1}$ D, Young Jun Choi ${ }^{2}$ iD, Yong Seok Lee ${ }^{3}$, and Yoo-Sam Chung ${ }^{1}$ \\ Departments of ${ }^{1}$ Otorhinolaryngology-Head and Neck Surgery, ${ }^{2}$ Radiology and Research Institute of Radiology, and ${ }^{3}$ Neurology, \\ Asan Medical Center, University of Ulsan College of Medicine, Seoul, Korea
}

\author{
한국 남성 수면무호흡 환자에서 설동맥과 혀의 위치관계 분석을 통한 \\ 혀 기저부 기능 수술 시 안전 영역에 대한 연구 \\ 박만준 ${ }^{1}$ 최영준 ${ }^{2} \cdot$ 이용석 ${ }^{3} \cdot$ 정유삼 $^{1}$ \\ 울산대학교 의과대학 서울아산병원 ${ }^{1}$ 이비인후과학교실, ${ }^{2}$ 영상의학교실, ${ }^{3}$ 신경과학교실
}

\author{
Received June 2, 2021 \\ Revised July 27,2021 \\ Accepted August 2, 2021 \\ Address for correspondence \\ Yoo-Sam Chung, MD, PhD \\ Department of Otorhinolaryngology- \\ Head and Neck Surgery, \\ Asan Medical Center, \\ University of Ulsan \\ College of Medicine, \\ 88 Olympic-ro 43-gil, Songpa-gu, \\ Seoul 05505, Korea \\ Tel +82-2-3010-3716 \\ Fax +82-2-489-2773 \\ E-mail yschung@amc.seoul.kr
}

Background and Objectives Upon operation of the tongue base for obstructive sleep apnea syndrome (OSAS), the lingual artery and the hypoglossal nerve are put at risk of injury, resulting in fatal complications such as massive bleeding upon damage. We studied the course of lingual artery in its relation with the tongue in OSAS patients and compared it with the ageand-gender-matched normal population.

Subjects and Method Korean male patients confirmed with OSAS by polysomnography, including those who had contrast-enhanced computed tomography (ceCT) of the head and neck, were defined as "OSAS group." Patients who had their ceCT image during the same study period were defined as the "control group." The control group was paired with the OSAS group by age and sex. By using foramen cecum (FC) as the main reference point, four reference marks were defined. For each reference point, the distance between both lingual arteries and the depth of the lingual artery from the lingual surface of the tongue were evaluated.

Results The depth of the lingual artery from the lingual surface of the tongue in the OSAS group $(25.1 \pm 8.6 \mathrm{~mm})$ was significantly different from the control $(29.5 \pm 5.9 \mathrm{~mm})$ at $1 \mathrm{~cm}$ anterior to the FC level $(p<0.014)$. The width of both lingual arteries was narrower in the OSAS group $(20.9 \pm 2.9)$ than in the control $(24.3 \pm 6.1)$ at the FC level $(p<0.003)$.

Conclusion The course of lingual artery and its spatial relation with the tongue in Korean male OSAS patients differs from the matched normal population.

Korean J Otorhinolaryngol-Head Neck Surg 2021;64(9):641-51

Keywords Hypoglossal nerve; Obstructive sleep apnea.

\section{Introduction}

The human upper airway is consisted with four anatomical levels; nasopharynx, velopharynx, glossopharynx, and laryn- gopharynx. ${ }^{1)}$ Majority of obstructive sleep apnea syndrome (OSAS) patients are previously studied to have a narrowing of glossopharynx and hypopharynx. Uvulopalatopharyngoplasty (UPPP) is a widely accepted first-line surgical treat-

This is an Open Access article distributed under the terms of the Creative Commons Attribution Non-Commercial License (https://creativecommons.org/licenses/by-nc/4.0) which permits unrestricted non-commercial use, distribution, and reproduction in any medium, provided the original work is properly cited. 
ment method for resolving OSAS. ${ }^{2)}$ However, in cases which UPPP fails to resolve OSAS, hypopharyngeal obstruction is accused to be the main reason. ${ }^{3)}$ Therefore, the importance of manipulation of the base of tongue (BOT) is currently being issued. ${ }^{4-6)}$ For the reduction of glossopharyngeal narrowing, a midline glossectomy could be an option. ${ }^{5)}$ In cases which hypopharyngeal narrowing is a problem, manipulation of BOT such as submucosal minimally invasive lingual excision, carbon dioxide laser midline glossectomy, hyoepiglottoplasty, and radiofrequency tissue reduction are accepted methods. ${ }^{4,5,7)}$ In combination with or without UPPP, the effectiveness of BOT manipulation techniques has been proven in many previous studies. $^{5,7)}$

In performing volumetric reduction in BOT, the hypoglossal/lingual artery neurovascular bundle (HLNVB) acts as a crucial anatomical hurdle. ${ }^{8)}$ The lingual arteries are the most vulnerable structure among HLNVB upon performing BOT manipulation, which may result in fatal complications such as massive bleeding and immediate airway obstruction when injured. ${ }^{9)}$ Many previous studies have focused on the course of the lingual artery and its spatial relation between adjacent anatomical landmarks. ${ }^{6,10-13)}$ However, there were no single previous study discussing the difference of lingual artery and its relation with the tongue in OSAS patients compared with the normal population.

In Korea, no previous study arguing about the spatial relation between the lingual artery and the tongue in Korean population had ever been reported. With the necessity, the authors aimed to analyze the course of the lingual artery and its relation with the tongue in Korean male adult population.

\section{Subjects and Methods}

\section{Ethics statement}

This study was approved by the Institutional Review Board of Asan Medical Center (Approval no. 2016-0348).

\section{Patient (OSAS) group}

From 2001 January to 2015 December, patients confirmed with OSAS by polysomnograph (PSG) in Asan Medical Center who underwent contrast-enhanced computed tomography (ceCT) or computed tomography angiography (CTA) of head and neck area for any occasion within one year prior or after PSG was included. Medical records, PSG data, and CT image of included patients were retrospectively reviewed.

\section{Control group}

The course of lingual artery and its spatial relation with the tongue were compared with the control group. Patients who had their ceCT or CTA image during the same study period mentioned above for any occasion (e.g.; acute tonsilitis, acute epiglottis, and etc.) were recruited, followed by randomized matching with the study group in terms of the age and the sex.

\section{Exclusion criteria}

In both OSAS group and control group, patients were excluded if they met the following criteria; 1) CT image of head and neck obtained from different protocols, 2) prior surgical history of naso-oro-hypo-pharyngeal area (e.g.; adenoidectomy, tonsillectomy, and etc.), 3) expected disruption of the natural course of the lingual artery (e.g.; abscess, tumors of pharyngeal region, and etc.), 4) female patients, and 5) patients under age of less than 20 years old.

The age was stratified upon every 10 years; the severity of obesity was classified according to the WHO 2000 guideline for Asia-Pacific population; ${ }^{14)}$ the OSAS severity was stratified upon apnea-hypopnea index (AHI), as described in the 2015 American Academy of Sleep Medicine recommendations. ${ }^{15)}$

\section{Imaging modality}

All OSAS patients and control subjects enrolled in the study have either received ceCT or CTA of head and neck area in the study period mentioned above.

CeCT was delivered by using a LightSpeed QX/i scanner (GE Medical Systems, Milwaukee, WI, USA) or Somatom Sensation 16 (Siemens Medical Solutions, Forchheim, Germany), and intravenous (IV) bolus injection of a nonionic iodinated contrast agent (140 mL) (Optiray; Mallinckrodt Pharmaceuticals, Dublin, Ireland) at a rate of $2.5 \mathrm{~mL} / \mathrm{s}$. Scanning was conducted using an $3 \mathrm{~mm}$-thick sections, and a 20.9-cm field of view, $120-\mathrm{kV}$ voltage, 200-mA tube current, and $256 \times 256$ matrix. Contiguous scans with $0.6 \mathrm{~mm}$ collimation were obtained at $3 \mathrm{~mm}$ intervals with no gap from the skull base to the upper chest for head and neck images. There were $70 \mathrm{sec}-$ onds delay of the scan phase following IV contrast infusion, thereby enabling the identification of the arterial and the venous system of head and neck as well as the course of the lingual artery.

CTA was performed through Somatom Sensation 16. IV line was secured at the patients' right arm, followed by a dual bolus IV infusion with a nonionic iodinated contrast media $(60 \mathrm{~mL})$ (Optiray) at a rate of $5 \mathrm{~mL} / \mathrm{s}$, in addition to saline in- 
fusion at a rate of $5 \mathrm{~mL} / \mathrm{s}$ at the same time. Scanning was conducted using a $3 \mathrm{~mm}$-thick sections, and a 20.9-cm field of view, $120-\mathrm{kV}$ voltage, $200-\mathrm{mA}$ tube current, and $256 \times 256$ matrix. Contiguous scans with $0.6 \mathrm{~mm}$ collimation were obtained at $3 \mathrm{~mm}$ intervals with no gap from the aortic arch to the vertex. Time delay from IV infusion to spiral scan was 14 seconds (monitoring delay; 10 seconds, followed by scan delay; 4 seconds). A Housefield unit of 100 was considered as arterial structure for the image reconstruction, thereby differentiating lingual artery from adjacent structures.

\section{Metric evaluation of the tongue \& the lingual artery}

For the measurement of each distance parameters, four landmarks were set as the reference points. The foramen cecum (FC) linguae, a small midline depression on the lingual surface on the border dividing the anterior two-thirds (tongue tip and tongue body) of the tongue and the posterior aspect (base of the tongue) of the tongue, was set as the main landmark in the study. In the sagittal image shown in the obtained CT scan, the dimpling point on the border separating the tongue body and BOT was identified, and defined as "point C" (Fig. 1). Three additional landmarks were defined as following detail; "point A," located $2 \mathrm{~cm}$ anteriorly from point $\mathrm{C}$ on the lingual surface; "point B," located $1 \mathrm{~cm}$ anteriorly from point $\mathrm{C}$ on

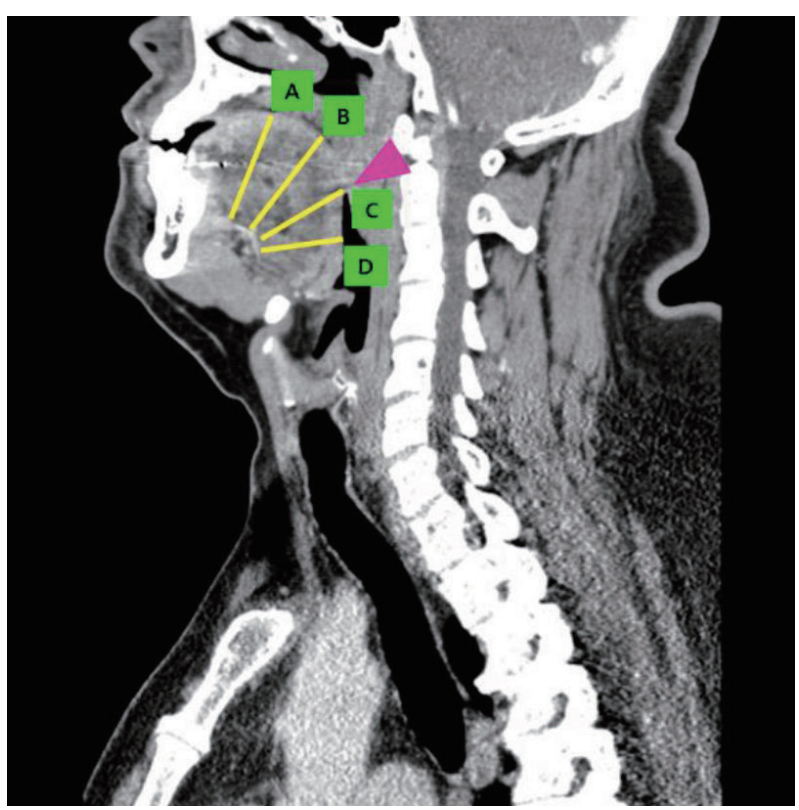

Fig. 1. Establishment of the reference points. A contract-enhanced computed tomography sagittal image of a 33-year-old male patient confirmed with obstructive sleep apnea syndrome. The lingual artery is visualized, and the depth of the lingual artery from the lingual surface of the tongue at each four reference points $(A-D)$ was measured. Arrowhead; FC. A: $2 \mathrm{~cm}$ anterior to FC. B: $1 \mathrm{~cm}$ anterior to FC. C: FC. D: $1 \mathrm{~cm}$ posterior to FC. FC, foramen cecum. the lingual surface; "point D" located $1 \mathrm{~cm}$ posteriorly from point $\mathrm{C}$ on the lingual surface. In all four points (point $\mathrm{A}-\mathrm{D}$ ), the depth of the lingual artery from the lingual surface of the tongue was measured on the sagittal image, and the width of lingual arteries bilaterally was measured on the axial image (Fig. 2). In the middle anterior half portion of the tongue, the depth and the width of deep lingual artery was measured, and in the posterior half of the tongue, the depth and the width of lingual artery was evaluated. The thickness, width, and length of the tongue were also measured (Fig. 3). Tongue thickness was measured as the distance from the lingual surface at FC level and mental spine on a sagittal image. The maximal width of the tongue shown on a coronal image was considered as the width of the tongue. The distance between the tip and the base of the tongue on a sagittal image was considered as the length of the tongue. All of the measurement process was done by a single specialist (M.J. Park), with the blinding of patients' clinical information.

\section{PSG}

Confirmation of OSAS was achieved based on full, standard PSG, delivered by RemLogic version 2.0 (Embla Systems Inc., Broomfield, CO, USA). A type 1 (standard, in-laboratory, technician-attended, overnight) protocol was employed. OSA was diagnosed when a patient had an AHI greater than 5 and symptoms of excessive daytime sleepiness, or an AHI greater than 15 regardless of daytime symptoms, in line with the 2015 American Academy of Sleep Medicine recommendations. ${ }^{15)}$ The severity of OSA was judged from AHI data, and graded as mild OSA $(5 \leq \mathrm{AHI}<15 / \mathrm{hr})$, moderate OSA $(15 \leq \mathrm{AHI}<30$ / $\mathrm{hr}$ ), and severe OSA (AHI $>30 / \mathrm{hr})$.

\section{Statistical analysis}

Statistical analysis was performed using SPSS version 22 software (IBM Corp., Armonk, NY, USA). Comparison of demographical features and distance parameters measured at each four reference points between the OSAS and the control group was done by using the student's t-test. Analysis of variance method was used for the analysis for the difference of each distance parameters in the subgroups of OSAS patients categorized by their obesity level, degree of OSAS, and the age. A Kolmogorov-Smirnov test was applied to determine whether each parameter showed a normal distribution. A $p$ value less than 0.05 was considered as statistically significant. 


\section{Results}

\section{Clinical and demographic characteristics of subjects}

Total numbers of 28 male patients have met inclusion criteria for the OSAS group during 2001 to 2015. In OSAS group, 4 (14.3\%) patients had CTA and 24 (85.7\%) patients had ceCT of head and neck area. Additionally, age-matched 28 male patients who had their CTA or ceCT taken during the same period were randomly recruited for the control group. In the control group, all the subjects had their ceCT taken (100\%), and no CTA was taken ( $0 \%$ ). The mean age of OSAS group was $49.86 \pm 12.74(\min 23, \max 74)$ years and the control group showed 49.96 \pm 12.99 ( $\min 24$, $\max 76$ ), which showed no significant difference of age in both groups. All patients in the

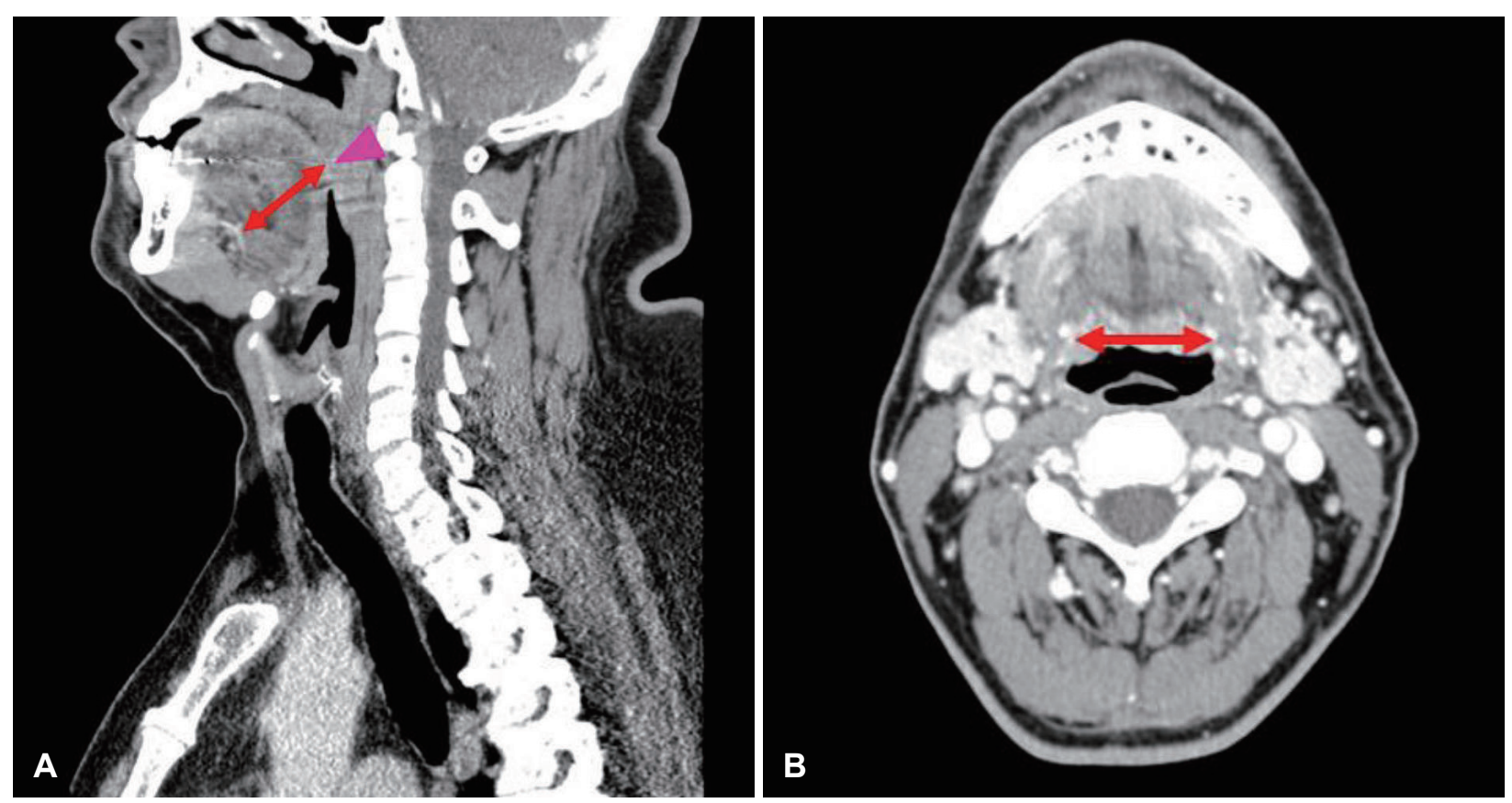

Fig. 2. Measuring the depth and the width of the lingual artery. A contract-enhanced computed tomography sagittal image of a 33-year-old male patient confirmed with obstructive sleep apnea syndrome. A: On the sagittal image, the depth of the lingual artery (arrowline) was measured from the lingual surface of the tongue at the FC level (arrowhead). B: The width of the bilateral lingual arteries was measured at the level of FC (arrowline). FC, foramen cecum.
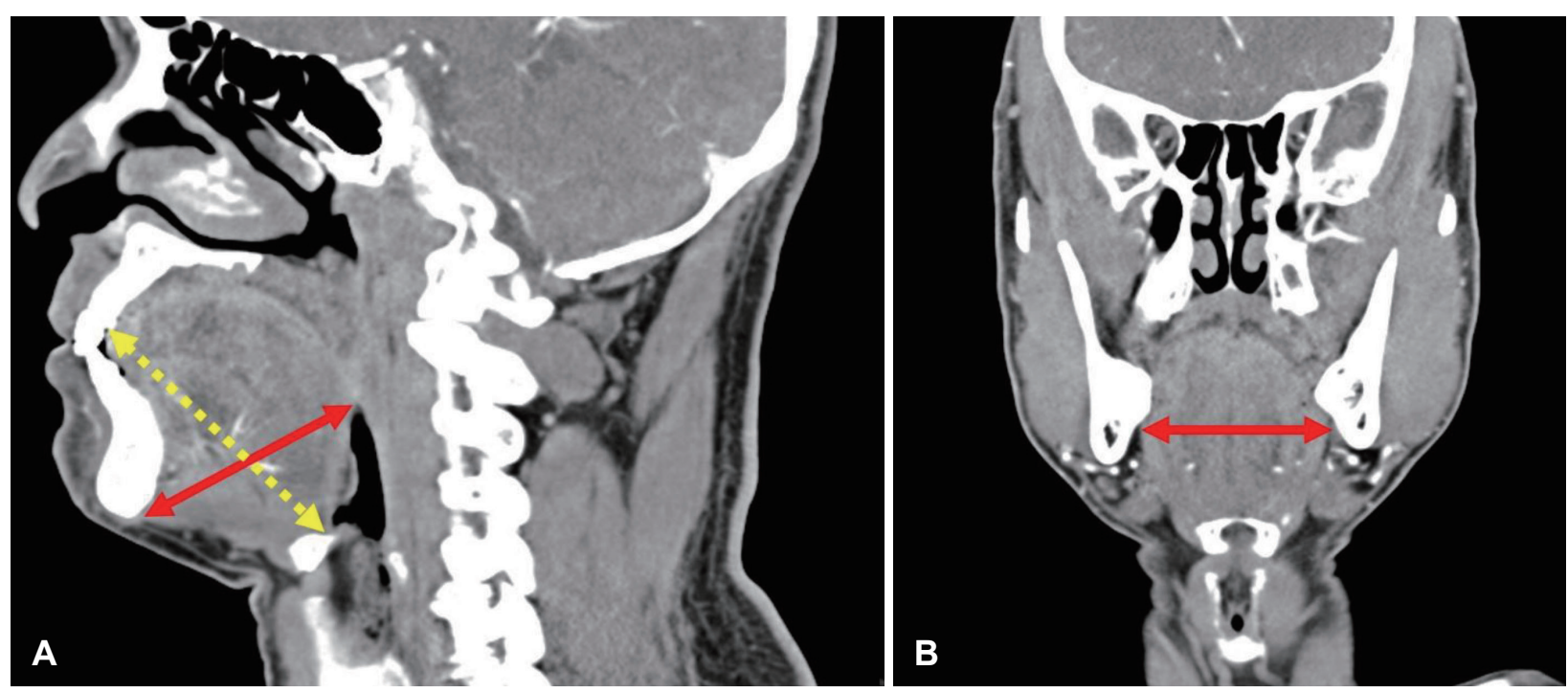

Fig. 3. Measurement of the thickness, width, and length of the tongue. A: Tongue thickness was measured as the distance from the lingual surface at foramen cecum level and mental spine on a sagittal image (bold line arrows). The distance between the tip and the base of the tongue on a sagittal image was considered as the length of the tongue (arrow with dotted lines). B: The maximal width of the tongue shown on a coronal image was considered as the width of the tongue. 
OSAS group and the control were male. The body mass index (BMI) of both OSAS group and control group are measured as following; $26.83 \pm 3.79(\min 20.90, \max 37.00)$ in the OSAS group, and $23.03 \pm 3.01$ ( $\min 16.42, \max 27.58$ ) in the control group, showing a significant higher BMI in the OSAS group with a $p$ value of less than 0.001 .

A full, standardized overnight PSG was done in all 28 patients in OSAS group, confirming the diagnosis of OSAS; (AHI $>5$ hours) in all 28 patients. In 28 OSAS patients, AHI showed $35.95 \pm 24.19$ ( $\min 8.50$, $\max 87.30)$, respiratory distress index of $38.60 \pm 22.68$ ( $\min 9.30, \max 89.20)$, minimal arterial oxyhemoglobin saturation $\left(\mathrm{SpO}_{2}\right)$ of $84.45 \pm 3.61$ (min 81.90, max 87.00), and mean $\mathrm{SpO}_{2}$ of $93.90 \pm 2.53(\min 84.20$, $\max 97.20)$.

\section{Metric evaluation of the tongue and its spatial relation with the lingual artery}

In OSAS group, the tongue was measured to be significant thicker in depth $(p=0.021)$, wider $(p=0.047)$, and longer $(p=$ $0.014)$ than in the control group. Contrary to the fact that no significant difference in the depth of the lingual artery at the point of FC were seen between OSAS group and control group, a difference in tongue thickness have resulted in significant difference in the relative depth of the lingual artery $(p<0.001)$ at the level of FC, indicating the lingual artery courses shallower in the OSAS group than the control group at the level of FC.

In all four reference points (A to D), the depth between the lingual artery and the tongue was measured, in addition to the

Table 1. Metric parameters of the tongue and the lingual artery in OSAS patients compared with normal population

\begin{tabular}{|c|c|c|c|c|c|c|}
\hline & \multicolumn{3}{|c|}{$\begin{array}{l}\text { Age and sex - matched } \\
\text { (total } 28 \text { pairs) }\end{array}$} & \multicolumn{3}{|c|}{ 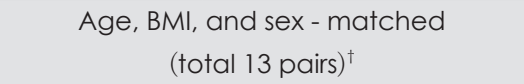 } \\
\hline & OSAS group & Control group & p-value & OSAS group & Control group & p-value \\
\hline \multicolumn{7}{|l|}{ Metric parameters of the tongue $(\mathrm{mm})$} \\
\hline Thickness & $\begin{array}{c}58.71 \pm 7.16 \\
(47.58-74.34)\end{array}$ & $\begin{array}{l}52.73 \pm 12.34 \\
(30.15-76.59)\end{array}$ & 0.021 & $\begin{array}{c}59.01 \pm 4.58 \\
(53.11-66.45)\end{array}$ & $\begin{array}{l}54.90 \pm 13.74 \\
(34.85-76.59)\end{array}$ & 0.345 \\
\hline Relative depth of the lingual artery* & $\begin{array}{l}0.52 \pm 0.06 \\
(0.41-0.62)\end{array}$ & $\begin{array}{l}0.62 \pm 0.11 \\
(0.46-0.79)\end{array}$ & $<0.001$ & $\begin{array}{l}0.52 \pm 0.03 \\
(0.48-0.58)\end{array}$ & $\begin{array}{l}0.62 \pm 0.11 \\
(0.46-0.79)\end{array}$ & 0.010 \\
\hline Width & $\begin{array}{c}55.45 \pm 6.53 \\
(42.96-66.43)\end{array}$ & $\begin{array}{c}52.97 \pm 1.60 \\
(50.68-55.76)\end{array}$ & 0.047 & $\begin{array}{c}53.81 \pm 5.21 \\
(42.96-61.31)\end{array}$ & $\begin{array}{c}52.32 \pm 1.14 \\
(50.68-54.74)\end{array}$ & 0.322 \\
\hline Length & $\begin{array}{c}74.90 \pm 3.84 \\
(67.22-81.15)\end{array}$ & $\begin{array}{c}72.63 \pm 3.11 \\
(67.50-77.54)\end{array}$ & 0.014 & $\begin{array}{c}74.74 \pm 3.74 \\
(42.96-80.89)\end{array}$ & $\begin{array}{c}73.14 \pm 3.32 \\
(68.50-77.54)\end{array}$ & 0.308 \\
\hline \multicolumn{7}{|c|}{ Depth of lingual artery at each reference points (distance from the lingual surface of the tongue) $(\mathrm{mm})$} \\
\hline Point $\mathrm{A}$ ( $2 \mathrm{~cm}$ anterior from $\mathrm{FC}$ ) & $\begin{array}{c}20.77 \pm 6.84 \\
(10.64-40.46)\end{array}$ & $\begin{array}{c}25.94 \pm 5.28 \\
(18.24-38.25)\end{array}$ & $<0.001$ & $\begin{array}{c}20.72 \pm 5.69 \\
(12.12-30.83)\end{array}$ & $\begin{array}{c}28.61 \pm 5.05 \\
(21.97-38.25)\end{array}$ & 0.002 \\
\hline Point B ( $1 \mathrm{~cm}$ anterior from FC) & $\begin{array}{l}25.06 \pm 8.55 \\
(8.68-47.17)\end{array}$ & $\begin{array}{c}29.46 \pm 5.86 \\
(19.50-44.70)\end{array}$ & 0.014 & $\begin{array}{c}23.81 \pm 5.64 \\
(11.95-30.12)\end{array}$ & $\begin{array}{c}31.80 \pm 6.36 \\
(25.22-44.70)\end{array}$ & 0.006 \\
\hline Point C (FC) & $\begin{array}{c}30.71 \pm 7.18 \\
(19.54-46.13)\end{array}$ & $\begin{array}{c}31.70 \pm 5.71 \\
(23.08-44.76)\end{array}$ & 0.243 & $\begin{array}{c}31.05 \pm 4.45 \\
(25.61-38.68)\end{array}$ & $\begin{array}{c}32.86 \pm 5.83 \\
(23.08-44.76)\end{array}$ & 0.378 \\
\hline Point D ( 1 cm posterior from FC) & $\begin{array}{c}33.26 \pm 5.55 \\
(22.33-47.72)\end{array}$ & $\begin{array}{c}34.78 \pm 6.80 \\
(11.16-43.68)\end{array}$ & 0.383 & $\begin{array}{c}33.78 \pm 4.73 \\
(27.70-42.45)\end{array}$ & $\begin{array}{c}35.26 \pm 4.59 \\
(27.29-43.68)\end{array}$ & 0.528 \\
\hline \multicolumn{7}{|c|}{ Width of lingual artery at each reference points (distance between the bilateral lingual arteries) $(\mathrm{mm})$} \\
\hline Point A (2 cm anterior from FC) & $\begin{array}{c}15.20 \pm 1.34 \\
(13.11-18.63)\end{array}$ & $\begin{array}{c}14.91 \pm 3.28 \\
(9.20-20.84)\end{array}$ & 0.324 & $\begin{array}{l}5.30 \pm 1.10 \\
(3.48-6.70)\end{array}$ & $\begin{array}{c}16.30 \pm 2.93 \\
(11.96-20.84)\end{array}$ & $<0.001$ \\
\hline Point B ( $1 \mathrm{~cm}$ anterior from FC) & $\begin{array}{c}17.29 \pm 1.95 \\
(13.69-22.26)\end{array}$ & $\begin{array}{c}20.72 \pm 4.01 \\
(13.17-29.33)\end{array}$ & $<0.001$ & $\begin{array}{c}7.55 \pm 1.51 \\
(5.24-10.75)\end{array}$ & $\begin{array}{c}21.80 \pm 3.41 \\
(14.39-29.33)\end{array}$ & $<0.001$ \\
\hline Point C (FC) & $\begin{array}{c}20.89 \pm 2.90 \\
(16.72-27.84)\end{array}$ & $\begin{array}{c}24.34 \pm 6.09 \\
(13.42-45.75)\end{array}$ & 0.003 & $\begin{array}{c}11.47 \pm 2.96 \\
(8.28-17.84)\end{array}$ & $\begin{array}{c}31.08 \pm 7.05 \\
(22.73-50.75)\end{array}$ & $<0.001$ \\
\hline Point $\mathrm{D}(1 \mathrm{~cm}$ posterior from $\mathrm{FC})$ & $\begin{array}{c}22.72 \pm 2.66 \\
(16.89-28.18)\end{array}$ & $\begin{array}{c}31.10 \pm 4.22 \\
(23.12-38.57)\end{array}$ & $<0.001$ & $\begin{array}{c}13.55 \pm 2.20 \\
(10.68-18.18)\end{array}$ & $\begin{array}{c}36.24 \pm 4.59 \\
(28.12-43.12)\end{array}$ & $<0.001$ \\
\hline
\end{tabular}

Data are presented as mean \pm standard deviation (min-max). *the relative depth of the lingual artery was measured as following, the depth of the lingual artery measured at level of FC divided by the depth of the tongue measured as the length between the mandible spine and the lingual surface of the tongue at the level of FC; tthe BMI was stratified according to the WHO criteria for the Asian population 2000, there were 13 numbers of subjects who have matched in the same age and BMI classification level, comparison of each metric parameters between two matched groups were done. OSAS, obstructive sleep apnea syndrome; FC, foramen cecum 
measurement of the distance between the bilateral lingual arteries. As shown in Table 1, the OSAS group showed significantly different depth below the lingual surface at point $\mathrm{A}$ and $\mathrm{B}$, whereas no significant difference depth was shown in point $\mathrm{C}$ and $\mathrm{D}$. The distance between lingual arteries on both sides showed no significant difference in point A in both groups, however OSAS group showed significantly different width of bilateral lingual arteries than the control group at point B, $\mathrm{C}$, and $\mathrm{D}(p<0.05)$. When the level of BMI was also matched in addition to the age with the control in 13 subjects, there were no significant differences in the thickness, width, and the length of the tongue, however the relative depth of the lingual artery showed significantly different depth in OSAS group. In both age and BMI matched party, the differences in the width of bilateral lingual arteries have widened with increased statistical significance, however no changes of the depth of the lingual artery were observed when only age was matched. A schematic illustration of the lingual artery and the tongue in OSAS group and control group in sagittal and axial view is shown in Fig. 4.

The measured distance parameters were analyzed upon obesity level, age, and severity of OSAS (Table 2). The severity of obesity was classified upon WHO classification 2000 for the Asian-pacific population, and the age was categorized upon three groups (age 20 to 39, 40 to 59, and 60 to 79). In OSAS group, there were no significant differences of the depth and the width in all four landmarks by the severity of obesity, OSAS, and age. In the control group, no significant differences of met- ric parameters were shown in all four landmarks by the age and severity of obesity, except in point A, a tendency for the wider and deeper course of the lingual artery was observed by the increase in BMI $(p<0.05)$.

\section{Discussion}

Currently, many surgeons accept surgical approach for the correction OSAS when a conservative method (e.g.; weight loss, continuous positive airway pressure device, and etc.) have failed to achieve therapeutic target. ${ }^{16)}$ UPPP is a powerful surgical method to resolve velopharyngeal obstruction, however has its demerit in cases which glossopharyngeal obstruction plays main role for the upper airway narrowing. ${ }^{2,3)}$ Manipulation of BOT in combination with UPPP or exclusively is an effective surgical modality for resolving hypopharyngeal narrowing, having its efficacies proven in many previous studies. ${ }^{4,5,7)}$ When manipulating the BOT, protection of the lingual artery as well as other components in HLNVB should come as the first priority as well as effective volumetric reduction of the BOT in the same time. Injury to the lingual artery could result in serious complications such as perioperative bleeding, hematoma, profuse blood aspiration, and pseudoaneurysm thereby obstructing the upper and lower airway immediately after or during the surgery. ${ }^{6,8,10,17)}$ Therefore, the importance of precise understanding of the lingual artery precisely in OSAS patients cannot be overemphasized for the surgeon.

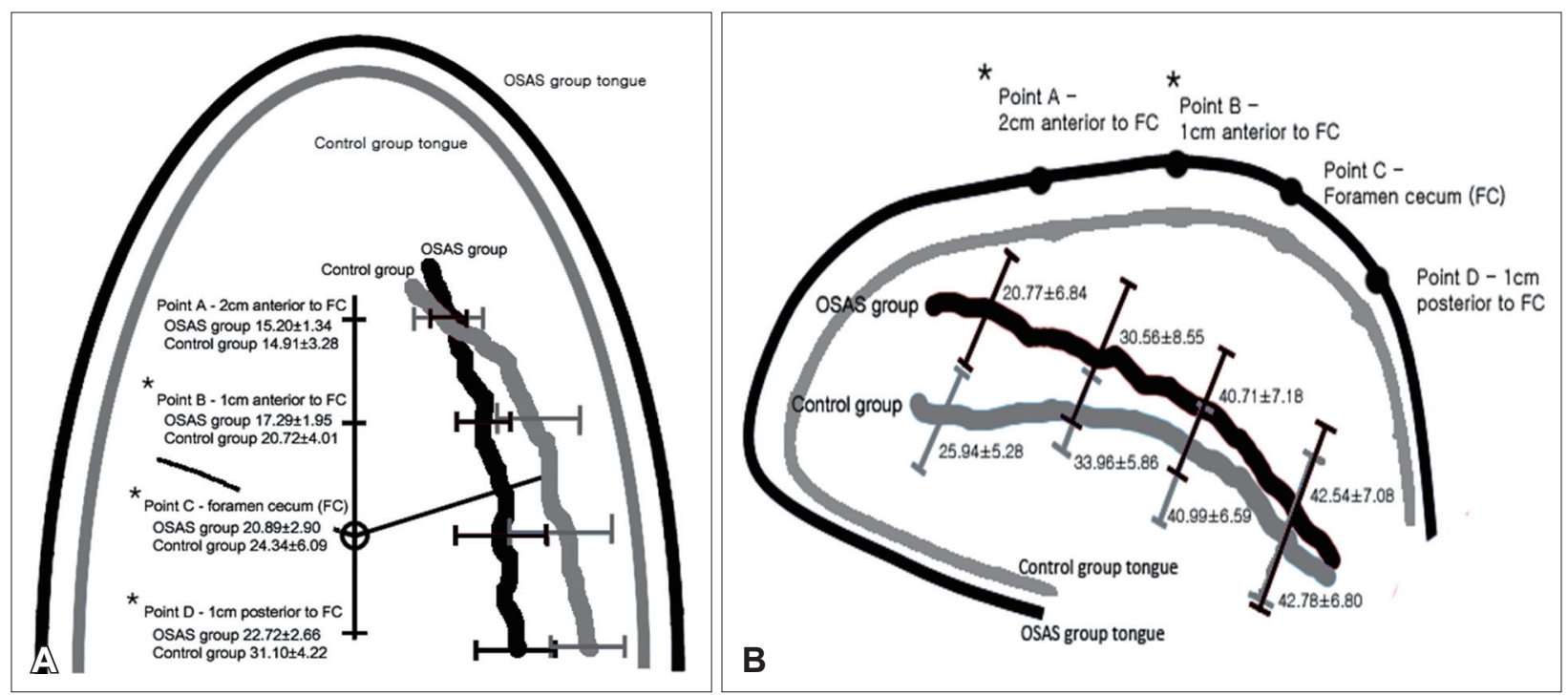

Fig. 4. A schematic illustration of anatomical difference between age-matched OSAS group and the control group. The average size of the tongue and the course of lingual artery in both OSAS (black lines) and age-sex matched control groups (gray lines) are drawn schematically (total 28 pairs). A: Viewed from the lingual surface. B: Sagittal view. The numbers represent the distance of the lingual artery from the lateral margin (A) or the ventral surface (B) of the tongue. ${ }^{*} p<0.05$. FC, foramen cecum; OSAS, obstructive sleep apnea syndrome. 


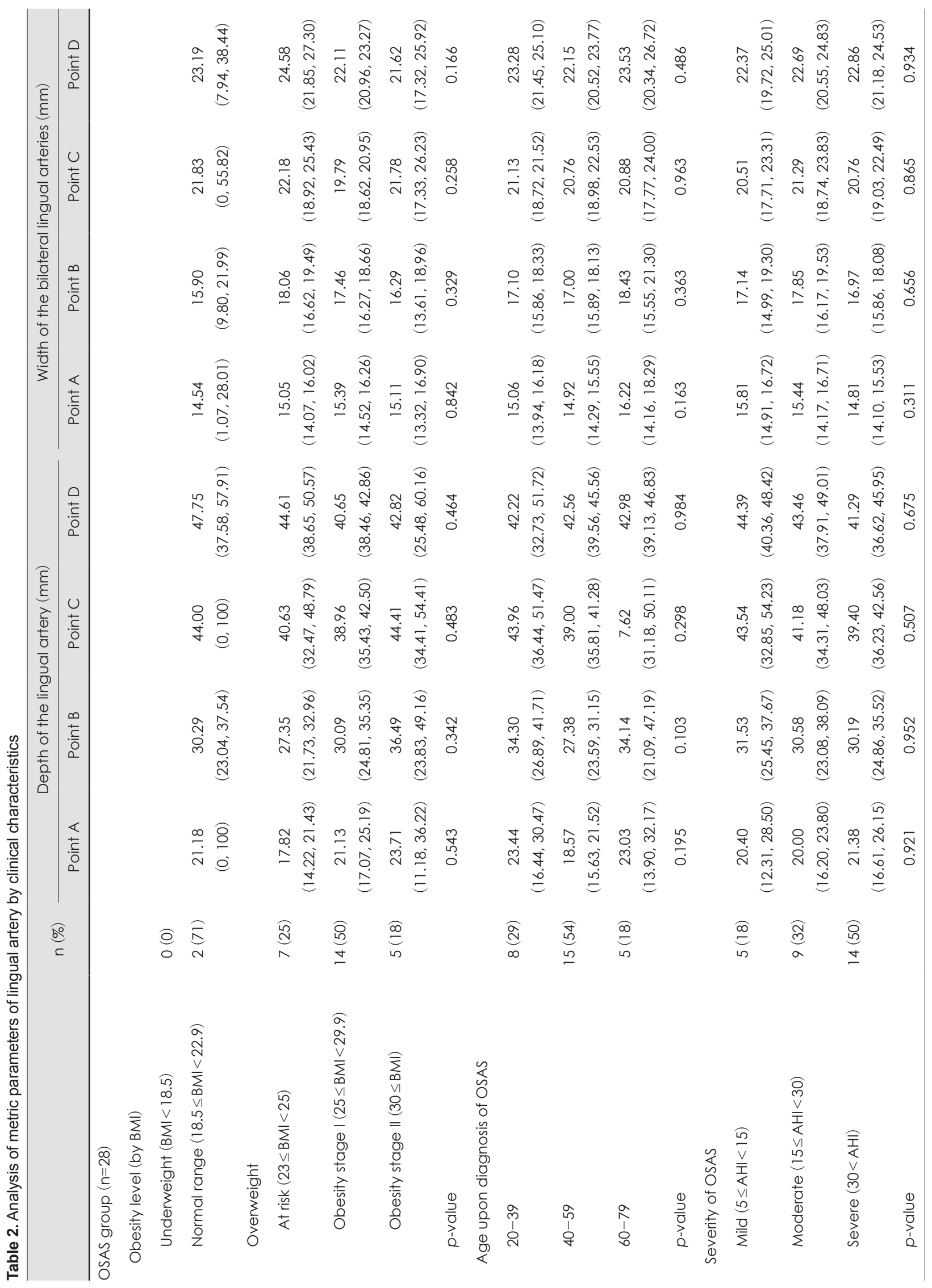




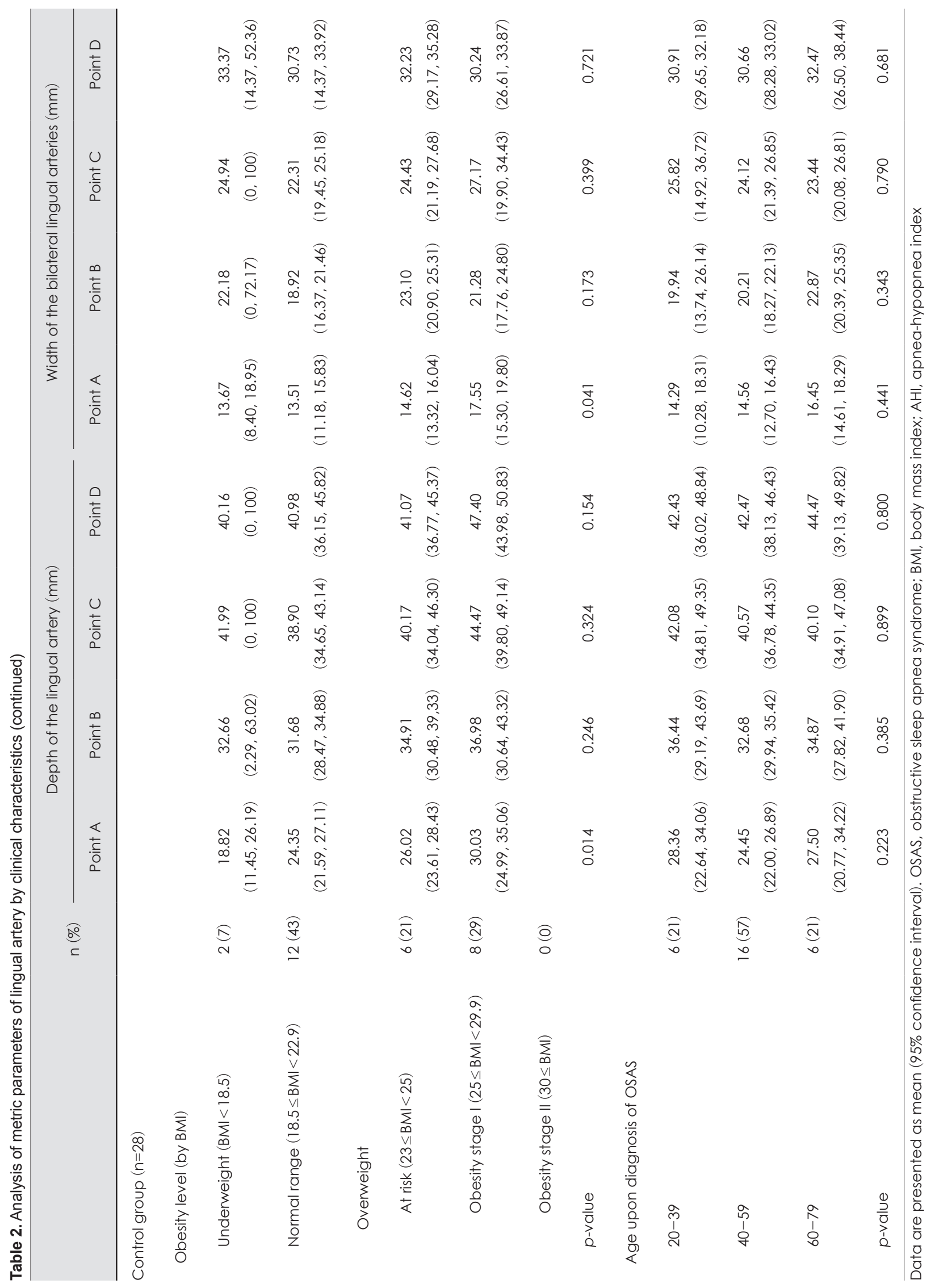


The lingual artery is a major artery branching out from the external carotid artery, and comes along as in the form of neurovascular bundle from the base of the tongue. HLNVB consists of lingual arteries, hypoglossal nerves, lingual nerves, and the lingual veins. ${ }^{8)}$ Posteriorly to circumvallate papillae, the lingual artery branches the dorsal lingual artery vertically towards to the dorsal surface of the tongue. At the level of the middle portion of the tongue, the lingual artery is divided into two minor arteries; the deep lingual artery located above the lingual frenulum; the sublingual artery located below the lingual frenulum, forming abundant vascular anastomosis on hypoglossal floor. ${ }^{18)}$ Lopez, et al. ${ }^{12)}$ have claimed three territories of the tongue by vascular supply; the dorsal portion supplied by the deep lingual artery; the ventral portion supplied by the sublingual artery; the basal portion supplied by the dorsal artery. When performing the functional surgery of the tongue, ventral portion (below lingual frenulum) are rarely manipulated, and the deep lingual artery is a relatively well reflected structure on the dorsal tongue. In contrary, the posterior to middle third portion of the tongue and the hypoglossal floor are considered as the "restrictive zone" when performing the functional surgery of the tongue, thereby making the dorsal lingual artery and post half of the lingual artery the most important structure among the course of the lingual artery. ${ }^{10,11)}$

Previously, attempts to identify the course of the lingual artery and its relations within the tongue were done by many studies. $^{8,12,13,18-21)}$ The lingual artery was identified in ceCT images and in cadavers, therefore the depth and the width of the both lingual arteries were reported by scholars in Turkey, China and North America. Hou, et al. ${ }^{11)}$ have proposed the "V zone" for the safe surgery of the tongue in OSAS patients, and $\mathrm{Wu}$, et al. ${ }^{13)}$ have analyzed the difference in the course of the lingual artery by the positional change of the tongue in Chinese population, and Cohen, et al. ${ }^{10)}$ and Ibrahim, et al. ${ }^{21)}$ have proven changes in the depth and the width of both lingual arteries by changes in the tongue position when retracted by a Feyh-Kastenbauer retractor, as if it was done in the setting for the transoral robotic surgery (TORS). Recently, Chang, et al. ${ }^{17)}$ prosed an intraoperative ultrasound imaging during TORS, which resulted in faster, safe, and more efficient surgical outcome. In 2021, Gualtieri, et al. ${ }^{20)}$ reported a new landmark on the ventral surface of the oropharynx to identify the lingual artery in the setting of a transoral surgery with resected head and neck specimen.

For the accurate comparison of the metric parameters between each study patients, a precise setting of each reference points was the single most important factor to consider upon study design. FC, located at the lingual surface of posterior one-third of the tongue, is an important landmark upon performing functional tongue surgeries. The feasibility of FC as a landmark for the evaluation of the lingual artery and the tongue had been validated by many studies over many decades. $^{6,11-13,18,20,21)}$ Therefore, we have set FC as the main reference point, and three additional reference points were set as $2 \mathrm{~cm}$ anteriorly, $1 \mathrm{~cm}$ anteriorly, and $1 \mathrm{~cm}$ posteriorly to $\mathrm{FC}$ on the lingual surface. The reason that we have set each additional reference points on the lingual surface of the tongue is for easier orientation upon performing the surgery of the tongue. Clinically, it is the lingual surface of the tongue, not the lingual artery itself viewed by the surgeon when it comes to the surgical manipulation of the tongue. ${ }^{20)}$ By identifying the FC on the lingual surface, the surgeon might easily get oriented of the spatial relation between the tongue and the lingual artery beneath the lingual surface, therefore lessening the rate of complications related to the damage of HLNVB. However, the exact location of FC is not easily identified on CT image, therefore we have set the FC as the point at onethird of the middle and posterior junction of the tongue in $\mathrm{CT}$ image exactly the same way as $\mathrm{Wu}$, et al. ${ }^{13)}$ have published.

Although the protocol for CT scan is different from the study conducted by $\mathrm{Wu}$, et al. ${ }^{13)}$ in China, we have compared our results side by side with their study. In patients confirmed with OSAS by PSG, both studies showed similar width of the lingual artery (20.9 mm in our study and $20.1 \mathrm{~mm}$ in Wu's study), however showed deeper course of the lingual artery in our study (30.7 $\mathrm{mm}$ in ours and $27.9 \mathrm{~mm}$ in Wu's) at the level of FC. The metric evaluation of the tongue showed slightly thicker tongue (58.7 $\mathrm{mm}$ in ours and $55.9 \mathrm{~mm}$ in Wu's), however shorter tongue (74.9 $\mathrm{mm}$ in ours and $83.5 \mathrm{~mm}$ in Wu's) in our study. A cadaveric study from China have revealed the safety margin for midline glossectomy as $7 \mathrm{~mm}$ lateral from the midline and $10 \mathrm{~mm}$ deep from the lingual surface at FC level. ${ }^{22)}$ However, it should be reminded that these results were delivered from cadavers, not OSAS patients who tend to be more obese, and the cadaveric tissues were fixed which makes fatty component have shrink, thereby altering the vascular structures within the tongue.

The abnormal craniofacial anatomy in adult OSAS patients compared with normal population were previously studied by many researchers over a long period. ${ }^{23-25)}$ Metric analysis on the narrowing of upper airway due to shortening of bony structures and enlarged oropharyngeal structures such as pal- 
atine tonsil, BOT, and the tongue itself on physical examination and CT image were previously reported in many studies. $^{23,24)}$ In addition, it had been argued patients with higher BMI tend to have more enlarged tongue with more abundant fat tissues in oropharyngeal structures, and these relationships were especially prevalent in Chinese population. ${ }^{19,25)}$ Similarly, our results showed the age-matched pairs of OSAS group had a thicker, wider, and longer length of the tongue compared with control, however these differences were no longer seen in BMI and age matched pairs (Table 1). Interestingly, the relative depth and width of the lingual arteries showed some significant differences in few reference points between the OSAS group and the control group. However, it should be considered that the OSAS group showed significant wider, thicker, and longer size of the tongue. Therefore, although some significant differences in the depth and the width of the lingual artery were shown in some reference points, it cannot be straightforwardly concluded that the course of the lingual artery is shallower in the anterior half of the tongue. Data shown in Table 2 shows no differences according to the level of obesity, age, and severity of OSAS were significantly related with the course of the lingual artery in both OSAS and control groups, supporting the fact that OSAS itself is an independent factor for shallower and narrower course of the lingual artery in the tongue, regardless of OSAS severity, age, and degree of obesity. The authors believe that these differences in the course of the lingual artery in the OSAS group and the control group are a result of the size difference of the tongue, however additional studies must be conducted to clear out this hypothesis.

Although we tried our best effort to minimize any bias and errors in the design of the study, we acknowledge some limitations as in following details. First, due to the fact that this study was a retrospective study, only one female OSAS patient was compatible for the inclusion criteria in the study period. To avoid any bias caused by the gender difference, we have excluded the female patient. Even though OSAS affects male population in Korea predominantly, it should be reminded that our data only represents the Korean male adult population. Second, in $\mathrm{Wu}^{13)}$ and Hou's ${ }^{11)}$ study, measurement of the lingual artery shown on CT image was done in a similar way with our study. In their study, CTA was performed in all subjects, exclusively enhancing only the arteries whereas we have recruited patients CTA in addition with ceCT of the head and neck area which shows not only arterial structures but venous structures as well. Anatomically, the lingual artery courses close with the lingual vein, forming the HLNVB. Upon per- forming the surgery of the tongue, not only the lingual artery but also the HLNVB itself should be secured from injury, therefore we planned to include patients with ceCT image as well. Third, there is no evidence that the control group in our study does not possess OSAS, since no PSG was performed on these patients. Fourth, the majority of the control group had acute infectious condition of the palatine tonsil and epiglottis. Considering the fact that acute infection of those area can alter in mucosal swelling of the tongue base, there is a possibility that the lingual artery depth in control group were under or overestimated. Finally, due to the fact that our study is a retrospectively designed study, we were not able to clarify the spatial relation of the lingual artery and the tongue during various positional changes of the tongue, as suggested in publication by Lu, et al. ${ }^{26)}$ Therefore, the authors acknowledge that the data provided in this paper is insufficient to provide a definite safety margin for the functional surgery in OSAS patients.

Despite some limitations, our study has its own unique clinical merits. First, sex and age-matched randomized normal population were recruited for the comparison. No significant difference of patients' age was observed, in contrary to the fact that a significantly higher BMI of OSAS group was evident than the control which indicates the randomization and the selection of control was successfully handled. Second, to avoid any possible measurement bias, the clinical characteristics of each subject were blinded upon measuring the metric parameters. Third, considering the fact that the numbers of patients who receive sleep surgeries are increasing yearly in Korea, establishing a comprehensive knowledge on the spatial relation between the lingual artery and the tongue in Korean male patients is in need. Since we are the first to report these findings in Korean population, we believe our study could act as a "cornerstone" in future clinical practice for many surgeons.

The course of lingual artery and the spatial relation between the tongue differs in Korean male OSAS patients compared with matched normal population. In OSAS patients, the lingual artery showed shallower depth anterior to FC, and the length between both lingual arteries narrower distance around the level of FC, compared to normal population. Age, degree of obesity and PSG derived parameters were not significantly related with the spatial relation between the tongue and the lingual artery. In practicing the functional surgery of the tongue, it should be considered that the "safety area" of the OSAS patients is different from the normal population. 


\section{Acknowledgments}

None.

\section{Author Contribution}

Conceptualization: all authors. Data curation: all authors. Formal analysis: Marn Joon Park, Yoo-Sam Chung. Investigation: Marn Joon Park, Yoo-Sam Chung. Methodology: Marn Joon Park, Young Jun Choi, Yoo-Sam Chung. Project administration: Marn Joon Park, Yoo-Sam Chung. Resources: all authors. Software: Marn Joon Park, Young Jun Choi, Yong Seok Lee. Supervision: Yoo-Sam Chung. Validation: Marn Joon Park, Young Jun Choi, Yoo-Sam Chung. Visualization: Marn Joon Park, Yoo-Sam Chung. Writing-original draft: Marn Joon Park. Writing-review \& editing: Marn Joon Park, Yoo-Sam Chung.

\section{ORCIDs}

Yoo-Sam Chung

Marn Joon Park

https://orcid.org/0000-0002-8866-5415

Young Jun Choi

https://orcid.org/0000-0003-1746-3266

https://orcid.org/0000-0001-7098-5042

\section{REFERENCES}

1) Boudewyns AN, Van de Heyning PH, De Backer WA. Site of upper airway obstruction in obstructive apnoea and influence of sleep stage. Eur Respir J 1997;10(11):2566-72.

2) Levin BC, Becker GD. Uvulopalatopharyngoplasty for snoring: Long-term results. Laryngoscope 1994;104(9):1150-2.

3) Farmer WC, Giudici SC. Site of airway collapse in obstructive sleep apnea after uvulopalatopharyngoplasty. Ann Otol Rhinol Laryngol 2000;109(6):581-4.

4) Friedman M, Soans R, Gurpinar B, Lin HC, Joseph N. Evaluation of submucosal minimally invasive lingual excision technique for treatment of obstructive sleep apnea/hypopnea syndrome. Otolaryngol Head Neck Surg 2008;139(3):378-84; discussion 385.

5) Hsieh TH, Fang TJ, Li HY, Lee SW. Simultaneous midline laser glossectomy with palatopharyngeal surgery for obstructive sleep apnoea syndrome. Int J Clin Pract 2005;59(4):501-3.

6) Li S, Shi H. Lingual artery CTA-guided midline partial glossectomy for treatment of obstructive sleep apnea hypopnea syndrome. Acta Otolaryngol 2013;133(7):749-54.

7) Sorrenti G, Piccin O, Mondini S, Ceroni AR. One-phase management of severe obstructive sleep apnea: Tongue base reduction with hyoepiglottoplasty plus uvulopalatopharyngoplasty. Otolaryngol Head Neck Surg 2006;135(6):906-10.

8) Lauretano AM, Li KK, Caradonna DS, Khosta RK, Fried MP. Anatomic location of the tongue base neurovascular bundle. Laryngoscope 1997;107(8):1057-9.

9) Herzog M, Schmidt A, Metz T, Günthner-Lengsfeld T, Bremert T, Hoppe F, et al. Pseudoaneurysm of the lingual artery after temperature-controlled radiofrequency tongue base reduction: A severe complication. Laryngoscope 2006;116(4):665-7.
10) Cohen DS, Low GM, Melkane AE, Mutchnick SA, Waxman JA, Patel S, et al. Establishing a danger zone: An anatomic study of the lingual artery in base of tongue surgery. Laryngoscope 2017; 127(1):110-5.

11) Hou T, Shao J, Fang S. The definition of the $V$ zone for the safety space of functional surgery of the tongue. Laryngoscope 2012; 122(1):66-70.

12) Lopez R, Lauwers F, Paoli JR, Boutault F, Guitard J. Vascular territories of the tongue: Anatomical study and clinical applications. Surg Radiol Anat 2007;29(3):239-44.

13) Wu D, Qin J, Guo X, Li S. Analysis of the difference in the course of the lingual arteries caused by tongue position change. Laryngoscope 2015;125(3):762-6.

14) Low $S$, Chin MC, Ma S, Heng D, Deurenberg-Yap M. Rationale for redefining obesity in Asians. Ann Acad Med Singap 2009;38(1): 66-9.

15) Ito E, Inoue Y. The international classification of sleep disorders, third edition. American Academy of Sleep Medicine. Includes bibliographies and index. Nihon Rinsho 2015;73(6):916-23.

16) Chirinos JA, Gurubhagavatula I, Teff K, Rader DJ, Wadden TA, Townsend R, et al. CPAP, weight loss, or both for obstructive sleep apnea. N Engl J Med 2014;370(24):2265-75.

17) Chang CC, Wu JL, Hsiao JR, Lin CY. Real-time, intraoperative, ultrasound-assisted transoral robotic surgery for obstructive sleep apnea. Laryngoscope 2021;131(4):E1383-90.

18) Abd-El-Malek S. Observations on the morphology of the human tongue. J Anat 1939;73(Pt 2):201-10.

19) Nashi N, Kang S, Barkdull GC, Lucas J, Davidson TM. Lingual fat at autopsy. Laryngoscope 2007;117(8):1467-73.

20) Gualtieri T, Verzeletti V, Ferrari M, Perotti P, Morello R, Taboni S, et al. A new landmark for lingual artery identification during transoral surgery: Anatomic-radiologic study. Head Neck 2021; 43(5):1487-98.

21) Ibrahim A, Kumar S, Patil AR, Aishwarya JG, Shah AS, Nair S. Radiological and cadaveric study of anatomical safe zone for transoral base of tongue surgery. J Robot Surg. In press 2020.

22) Ye J, Wang J, He L, Han D. Anatomic characteristics of lingual artery and midline glossectomy. Zhonghua Er Bi Yan Hou Ke Za Zhi 2001;36(1):55-7.

23) Bayat M, Shariati M, Rakhshan V, Abbasi M, Fateh A, Sobouti F, et al. Cephalometric risk factors of obstructive sleep apnea. Cranio 2017;35(5):321-6.

24) Cuccia AM, Campisi G, Cannavale R, Colella G. Obesity and craniofacial variables in subjects with obstructive sleep apnea syndrome: Comparisons of cephalometric values. Head Face Med 2007;3:41.

25) Yu X, Fujimoto K, Urushibata K, Matsuzawa Y, Kubo K. Cephalometric analysis in obese and nonobese patients with obstructive sleep apnea syndrome. Chest 2003;124(1):212-8.

26) Lu H, Qin J, Yue R, Liu C, Li S, Wu D. Application of 3D reconstruction for midline glossectomy in OSA patients. Eur Arch Otorhinolaryngol 2020;277(3):925-31. 